PROCEEDINGS OF THE

AMERICAN MATHEMATICAL SOCIETY

Volume 128, Number 7, Pages 2043-2049

S 0002-9939(99)05256-9

Article electronically published on November 1, 1999

\title{
ON THE DIMENSION FUNCTION OF ORTHONORMAL WAVELETS
}

\author{
MANOS PAPADAKIS
}

(Communicated by David R. Larson)

\begin{abstract}
We announce the following result: Every orthonormal wavelet of $L^{2}(\mathbf{R})$ is associated with a multiresolution analysis such that for the subspace $V_{0}$ the integral translates of a countable at most family of functions is a tight frame.
\end{abstract}

Orthonormal wavelets are square-integrable functions $\psi$ such that the set

$$
\left\{2^{j / 2} \psi\left(2^{j} x-k\right): j, k \in \mathbf{Z}\right\}
$$

is an orthonormal basis of $L^{2}(\mathbf{R})$.

Theorem 1. If a function $\psi$ in $L^{2}(\mathbf{R})$ is an orthonormal wavelet, then we have

$$
\sum_{k \in \mathbf{Z}}|\widehat{\psi}(\xi+2 k \pi)|^{2}=1 \quad \text { for } \quad \text { a.e. } \quad \xi \in \mathbf{R}
$$

and

$$
\sum_{k \in \mathbf{Z}} \widehat{\psi}\left(2^{j}(\xi+2 k \pi)\right) \overline{\widehat{\psi}(\xi+2 k \pi)}=0 \quad \text { for } \quad \text { a.e. } \quad \xi \in \mathbf{R}, \quad j \geq 1
$$

Let $W_{j}$ be the closed linear span of the set $\left\{2^{j / 2} \psi\left(2^{j} x-k\right): k \in \mathbf{Z}\right\}$. An orthonormal wavelet $\psi$ is associated with a multiresolution analysis (MRA) $\left\{V_{j}\right\}_{j}$ if the set $\{\psi(x-k): k \in \mathbf{Z}\}$ is an orthonormal basis of $V_{1} \cap V_{0}^{\perp}$. For a complete, rigorous and comprehensive introduction to wavelet analysis, the appropriate definitions and a complete proof of the previous theorem the reader may refer to 5 .

Journé gave the first example of an orthonormal wavelet not associated with an MRA. On the other hand Auscher proved (see 1]) that orthonormal wavelets (or simply wavelets) whose Fourier transforms satisfy a mild smoothness condition and a weak decay condition at $\infty$ are associated with an MRA.

Let

$$
D_{\psi}(\xi):=\sum_{j=1}^{\infty} \sum_{k}\left|\hat{\psi}\left(2^{j}(\xi+2 k \pi)\right)\right|^{2}, \quad \xi \in \mathbf{T}:=[-\pi, \pi) .
$$

Obviously, $D_{\psi}$ is a well-defined $2 \pi$ periodic integrable function called the dimension function first introduced by Auscher. Completely independently Gripenberg ([6]) and Wang ([7]) proved the following result:

Received by the editors June 15, 1998 and, in revised form, August 25, 1998.

1991 Mathematics Subject Classification. Primary 41A15, 41A30, 42A38, 42C15, 46 N99.

Key words and phrases. Multiresolution analysis, wavelets, dimension function, frames. 
Theorem 2. Let $\psi$ be an orthonormal wavelet. Then $\psi$ is associated with an MRA if and only if $D_{\psi}(\xi)=1$ a.e. in $\mathbf{T}$.

We generalize the previous theorem by proving that every orthonormal wavelet can be associated with a more general type of MRA generated by frames of integral translates of a countable at most family of functions. This new family of MRAs defined below generalizes the frame MRA introduced by Benedetto and Li ([2]). Let $D$ be the unitary operator called the Dilation operator defined by $D f(t)=\sqrt{2} f(2 t)$, $f \in L^{2}(\mathbf{R})$. In order to clarify our notation, we define the Fourier transform $\widehat{f}$ of $f \in L^{1}(\mathbf{R})$ by the following formula:

$$
\widehat{f}(\xi)=\int_{-\infty}^{\infty} f(t) e^{-i t \xi} d \xi
$$

Definition 3. A generalized frame multiresolution analysis (GFMRA) $\left\{V_{j}\right\}_{j}$ is an increasing chain of closed subspaces of $L^{2}(\mathbf{R})$ such that:

1. For every integer $j$ we have that $V_{j+1}=D\left(V_{j}\right)$.

2. $\bigcap_{j} V_{j}=\{0\}$ and the closure of $\bigcup_{j} V_{j}$ is $L^{2}(\mathbf{R})$.

3. There exist $\varphi_{i}, i \in L \subseteq \mathbf{Z}^{+}$, in $V_{0}$ such that the set $\left\{\varphi_{i}(x-k): i \in L\right.$, $k \in \mathbf{Z}\}$ is a frame for $V_{0}$.

We announce the following result:

Theorem 4. Let $\psi$ be an orthonormal wavelet. Then $\psi$ is associated with a GFMRA in the sense that $\{\psi(x-k): k \in \mathbf{Z}\}$ is an orthonormal basis of $V_{1} \cap V_{0}^{\perp}$.

The fact that the dimension function gives the dimension of a certain type of subspaces (see Lemma 5) motivated us to prove this result. Next we state two necessary lemmas for the proof of this theorem. We close with an application of the previous theorem to Journé's example.

Let $L^{2}\left(\mathbf{T}, l^{2}\right)$ be the Hilbert space of $l^{2}(\mathbf{Z})$-valued measurable functions defined on the torus with the inner product given by the equation

$$
\langle f, g\rangle=\int_{-\pi}^{\pi}\langle f(t), g(t)\rangle d t, \quad \quad f, g \in L^{2}\left(\mathbf{T}, l^{2}\right) .
$$

Let $X$ be the unitary operator mapping $L^{2}(\mathbf{R})$ onto $L^{2}\left(\mathbf{T}, l^{2}\right)$ defined by

$$
X f(t)=\{f(t+2 k \pi)\}_{k}, \quad t \in[-\pi, \pi) .
$$

Let $\Psi_{j}(\xi):=X\left(2^{-j / 2}\left(D^{-j} \psi\right)^{\wedge}\right)=\left\{\hat{\psi}\left(2^{j}(\xi+2 k \pi)\right)\right\}_{k}, j \geq 1$. It is easy to check that the following equations are true for every orthonormal wavelet $\psi$ :

$$
D_{\psi}(\xi)=\sum_{j=1}^{\infty}\left\|\Psi_{j}(\xi)\right\|^{2} \quad \text { and } \quad \int_{\mathbf{T}} D_{\psi}(\xi) d \xi=2 \pi .
$$

Therefore $D_{\psi}(\xi)$ is finite a.e.

Lemma 5 ([1]). For every $\xi \in \mathbf{T}$ such that $D_{\psi}(\xi)$ is finite, we have that $D_{\psi}(\xi)$ is the dimension of the (closed) subspace spanned by $\left\{\Psi_{j}(\xi): j \geq 1\right\}$.

The previous lemma is an immediate consequence of a more general result (see Proposition 5.2 in [1] or Lemma 3.7 in [5]). Because of this lemma, the function $D_{\psi}$ was called the dimension function and takes only integer values; therefore its range is at most countable. The second lemma we need is the following: 
Lemma 6. Let $v_{j}:=\left(v_{i j}\right)_{i \in \mathbf{Z}}, j=1,2, \ldots,(l+1)$, be elements of $l^{2}(\mathbf{Z})$. Assume that $v_{1}, v_{2}, \ldots, v_{l}$ are linearly independent. Then $v_{1}, v_{2}, \ldots, v_{l}, v_{l+1}$ are linearly dependent if and only if all the $(l+1) \times(l+1)$ matrices of the form $\left(v_{i j}\right)_{i \in I, j}$ have zero determinants, where $I$ is an arbitrary subset of $\mathbf{Z}$ with cardinality $l+1$.

This lemma suggests that if we have a set of $l$ linearly independent vectors of $l^{2}(\mathbf{Z})$, by adding a new vector, the new set of $l+1$ vectors is linearly independent if and only if one of the $(l+1) \times(l+1)$ determinants produced by choosing arbitrarily $(l+1)$ rows from the $\infty \times(l+1)$ matrix produced by the $l+1$ vectors is non-zero. The proof of this linear algebra lemma is not difficult and will be omited.

Proof of Theorem \& Assume that the range of $D_{\psi}$ is the set of non-negative integers $\mathcal{R}:=\left\{a_{i}: i \in L \cup\{0\}\right\}$, where $L$ is a subset of $\mathbf{Z}^{+}$. Since $\int_{-\pi}^{\pi} D_{\psi}(\xi) d \xi=2 \pi$, then if at least one of the values of $D_{\psi}$ is greater than 1 , we have $0 \in \mathcal{R}$. Otherwise $D_{\psi}(\xi)=1$ a.e., thus $\psi$ is associated with an MRA, so in this case the theorem is proved.

Therefore, assume $a_{0}=0$ and $\left\{a_{i}: i \in L\right\} \subseteq \mathbf{Z}^{+}$. Also assume that $a_{i}, i \in L$, are distinct integers. Let $I_{i}=\left\{\xi \in \mathbf{T}: D_{\psi}(\xi)=a_{i}\right\}$. Let us first focus on $I_{1}$. Define $f_{1}: I_{1} \rightarrow \mathbf{Z}^{+}$by the following formula:

$$
f_{1}(\xi):=\min \left\{j \in \mathbf{Z}^{+}: \Psi_{j}(\xi) \neq 0\right\} .
$$

Lemma 5 shows that such an integer $j$ exists for every $\xi \in I_{1}$, since $a_{1} \geq 1$. Now let $I_{1, j}:=f_{1}^{-1}(\{j\})$. Since $\Psi_{j}$ are measurable functions, the family $\left\{I_{1, j}: \quad j \geq 1\right\}$ is a measurable a.e. partition of $I_{1}$, i.e. $I_{1} \backslash \bigcup_{j=1}^{\infty} I_{1, j}$ is null. Notice that some of $I_{1, j}$ may be empty or null. Assume for the moment that $a_{1}>1$. Now, let $j_{1} \in \mathbf{Z}^{+}$ be such that $I_{1, j_{1}}$ is non-null. Since $\operatorname{dim}\left[\Psi_{j}(\xi): j \in \mathbf{Z}^{+}\right]^{-}=a_{1}>1$, for all $\xi \in I_{1, j_{1}}$ we can define

$$
f_{2}(\xi):=\min \left\{j>j_{1}: \Psi_{j_{1}}(\xi) \text { and } \Psi_{j}(\xi) \text { are linearly independent }\right\} .
$$

Now let $I_{1, j_{1}, j}:=f_{2}^{-1}(\{j\}), j>j_{1}$. Then we have that if $I_{1, j_{1}, j}$ is non-null, then $\xi$ belongs to $I_{1, j_{1}, j}$ if and only if $\Psi_{j_{1}}(\xi)$ and $\Psi_{j}(\xi)$ are linearly independent and the set $\left\{\Psi_{j_{1}}(\xi), \Psi_{l}(\xi): j_{1} \leq l<j\right\}$ is linearly dependent. We claim that $I_{1, j_{1}, j}$ is measurable.

Proof of the claim. Due to the definition of $I_{1, j_{1}, j}$ we have that $\Psi_{j_{1}}(\xi)\left(\xi \in I_{1, j_{1}, j}\right)$ is non-zero and $\left\{\Psi_{j_{1}}(\xi), \Psi_{j}(\xi)\right\}$ is a linearly independent set of vectors. Therefore by Lemma 6 there exists a $2 \times 2$ submatrix of the $\infty \times 2$ matrix with columns the vectors $\Psi_{j_{1}}(\xi)$ and $\Psi_{j}(\xi)$ with non-zero determinant. On the other hand for every integer $l$ such that $j_{1} \leq l<j$ and $\xi \in I_{1, j_{1}, j}$ that set of vectors $\left\{\Psi_{j_{1}}(\xi), \Psi_{l}(\xi)\right\}$ is linearly dependent; thus the determinants of all $2 \times 2$ submatrices of the $\infty \times 2$ matrix with columns the vectors $\Psi_{j_{1}}(\xi)$ and $\Psi_{l}(\xi)$ vanish. The previous argument and the fact that determinants of measurable functions are measurable themselves imply that $I_{1, j_{1}, j}$ is measurable.

It is not hard to see that the family $\left\{I_{1, j_{1}, j}: j>j_{1}\right\}$ is an a.e. partition of $I_{1, j_{1}}$. Repeating this process we can define a measurable a.e. partition $\left\{I_{1, \mathbf{j}}: \mathbf{j} \in\left(\mathbf{Z}^{+}\right)^{a_{1}}\right\}$ of $I_{1}$, such that for every $\xi$ in a non-null $I_{1, \mathbf{j}}$, where $\mathbf{j}=\left(j_{1}, j_{2}, \ldots, j_{a_{1}}\right)$, we have that the set $\left\{\Psi_{j_{1}}(\xi), \Psi_{j_{2}}(\xi), \ldots, \Psi_{j_{a_{1}}}(\xi)\right\}$ is a linearly independent set.

Since for every such $\xi$ the dimension of the subspace $\left[\Psi_{j}(\xi): j \in \mathbf{Z}^{+}\right]^{-}$is equal to $a_{1}$ we obtain that the previous set is a linear basis of the subspace $\left[\Psi_{j}(\xi): j \in \mathbf{Z}^{+}\right]^{-}$. Applying the Gram-Schmidt orthogonalization process we conclude that there exist 
$\Phi_{1,1}, \Phi_{1,2}, \ldots, \Phi_{1, a_{1}}: I_{1} \rightarrow \ell^{2}(\mathbf{Z})$ measurable functions such that for every $\xi \in I_{1}$ the set $\left\{\Phi_{1,1}(\xi), \Phi_{1,2}(\xi), \ldots \Phi_{1, a_{1}}(\xi)\right\}$ is an orthonormal basis of $\left[\Psi_{j}(\xi): j \in \mathbf{Z}^{+}\right]^{-}$. The same arguments apply for the remaining sets $I_{i}$; thus we obtain measurable functions $\Phi_{i, k}, i \in L, k=1,2, \ldots, a_{i}$, sharing the same properties with $\Phi_{1, k}$. For notational purposes we use $e^{i n}$. to denote the function $\xi \rightarrow e^{i n \xi}$.

Claim. For every $i \in L$ the set $\left\{e^{i n .} \Phi_{i, k}: k=1,2, \ldots, a_{i}, \quad n \in \mathbf{Z}\right\}$ is a tight frame of its closed linear span with frame bound equal to $2 \pi$ contained in the subspace $L^{2}\left(I_{i}, l^{2}\right)$.

Proof of the claim. Let $L^{2}\left(I_{i}\right)$ be the closed subspace of $L^{2}(\mathbf{T})$ containing all functions supported on $I_{i}$ and $A_{i, k}$ be the bounded operator defined by

$$
A_{i, k}(u):=u \Phi_{i, k}, \quad u \in L^{2}\left(I_{i}\right) .
$$

It is not hard to verify that $A_{i, k}$ is an isometry, since $\left\|\Phi_{i, k}(\xi)\right\|=1$ for a.e. $\xi \in I_{i}$. Let $\chi_{I_{i}}$ be the characteristic function of $I_{i}$. It is also easy to check that the set $\left\{\frac{1}{\sqrt{2 \pi}} \chi_{I_{i}} e^{i n .}: n \in \mathbf{Z}\right\}$ is a tight frame for $L^{2}\left(I_{i}\right)$ with frame constant 1 (this is a partial case of a more general result studied in [4]). This proves the claim.

Since for a.e. $\xi \in I_{i}$ the set $\left\{\Phi_{i, k}(\xi): k=1,2, \ldots, a_{i}\right\}$ is an orthonormal basis for $\left[\Psi_{j}(\xi): j \in \mathbf{Z}^{+}\right]^{-}$and $\left|I_{i} \cap I_{j}\right|=0$ for $i \neq j, i, j \in L$, we can easily verify that the set $\bigcup_{i \in L} \bigcup_{k=1}^{a_{i}}\left\{e^{i n .} \Phi_{i, k}: n \in \mathbf{Z}\right\}$ is a tight frame of its closed linear span, which we denote by $\widetilde{V_{0}}$, with the same frame bound.

Next we will prove that $X\left(\sum_{j \geq 1} \oplus \widehat{W_{-j}}\right) \subseteq \widetilde{V_{0}}$, where $\widehat{W_{-j}}$ is the image of $W_{-j}$ under the Fourier transform: First notice that for every $j \in \mathbf{Z}^{+}$and $l \in \mathbf{Z}$ we have

$$
e^{i 2^{j} l .} \Psi_{j}=\sum_{i \in L} \chi_{I_{i}} e^{i 2^{j} l \cdot} \Psi_{j}
$$

Pick $I_{i}$ with $i \in L$. Since for almost every $\xi$ in $I_{i}\left\{\Phi_{i, 1}(\xi), \Phi_{i, 2}(\xi), \ldots, \Phi_{i, a_{i}}(\xi)\right\}$ is an orthonormal basis of $\left[\Psi_{j}(\xi): j \in \mathbf{Z}^{+}\right]^{-}$we get that for every $j \in \mathbf{Z}^{+}$we have

$$
\Psi_{j}(\xi)=\sum_{k=1}^{a_{i}} \nu_{i, k}^{j}(\xi) \Phi_{i, k}(\xi)
$$

where $\nu_{i, k}^{j}(\xi)=\left\langle\Psi_{j}(\xi), \quad \Phi_{i, k}(\xi)\right\rangle$ and $\nu_{i, k}^{j}$ vanish outside $I_{i}$. Obviously $\nu_{i, k}^{j}$ are measurable functions and belong to $L^{2}(\mathbf{T})$. If $\nu_{i, k}^{j}(\xi)=\sum_{m} a_{i, k}^{j}(m) e^{-i m \xi}$, we obtain

$$
\chi_{I_{i}} \Psi_{j}=\sum_{k=1}^{a_{i}} \sum_{m} a_{i, k}^{j}(m) e^{-i m \cdot} \Phi_{i, k} .
$$

Due to Eq. (3), by multiplying both sides of the previous equation by $e^{-i 2^{j} l \xi}, l \in \mathbf{Z}$, we get that every element of the orthonormal basis $\left\{e^{-i 2^{j} l .} \Psi_{j}: j \in \mathbf{Z}^{+}, \quad l \in \mathbf{Z}\right\}$ of $X\left(\sum_{j \geq 1} \oplus \widehat{W_{-j}}\right)$ is contained in $\widetilde{V_{0}}$.

To complete the proof we must establish the converse inclusion. For every $\xi$ in a non-null $I_{i, \mathbf{j}}$ we can write each $\Phi_{i, k}(\xi)$ as a finite linear combination of the basis elements $\Psi_{j_{1}}(\xi), \Psi_{j_{2}}(\xi), \ldots, \Psi_{j_{a_{i}}}(\xi)$, where $\mathbf{j}=\left(j_{1}, j_{2}, \ldots, j_{a_{i}}\right)$. Therefore there exist measurable functions $\mu_{k, p}^{i}$ vanishing outside $I_{i, \mathbf{j}}$ such that

$$
\Phi_{i, k}(\xi)=\sum_{p=1}^{a_{i}} \mu_{k, p}^{i}(\xi) \Psi_{j_{p}}(\xi), \quad \xi \in I_{i, \mathbf{j}} .
$$


We extend $\mu_{k, p}^{i} 2 \pi$ periodicaly on the real line and define $\widehat{\phi_{i, k}}:=X^{-1}\left(\Phi_{i, k}\right)$. Now the previous equation yields

$$
\widehat{\phi_{i, k}}(\xi)=\sum_{p=1}^{a_{i}} \mu_{k, p}^{i}(\xi) \widehat{\psi}\left(2^{j_{p}} \xi\right) \quad \text { a.e. in } \mathbf{R} \text {. }
$$

Next we prove that all $\widehat{\phi_{i, k}}$ are perpendicular to $\widehat{\psi_{j, l}}$ for all $j \geq 0$ and $l \in \mathbf{Z}$ : Indeed,

$$
\begin{aligned}
\left\langle\widehat{\phi_{i, k}}, \quad \widehat{\psi_{j, k}}\right\rangle & =2^{-j / 2} \int_{-\infty}^{\infty} \widehat{\phi_{i, k}}(\xi) \widehat{\widehat{\psi}\left(2^{-j} \xi\right)} e^{i \frac{\xi}{2^{j}} l} d \xi \\
& =2^{j / 2} \int_{-\pi}^{\pi}\left[\sum_{n \in \mathbf{Z}} \widehat{\phi_{i, k}}\left(2^{j}(\xi+2 n \pi)\right) \overline{\widehat{\psi}(\xi+2 n \pi)}\right] e^{i \xi l} d \xi
\end{aligned}
$$

Due to the definition of $\phi_{i, k}$ we have that

$$
\operatorname{essup}\left\{\sum_{n \in \mathbf{Z}}\left|\widehat{\phi_{i, k}}(\xi+2 n \pi)\right|^{2}: \xi \in \mathbf{T}\right\} \leq 1 .
$$

Combining this fact with Eq. (1) we obtain that the sum

$$
\sum_{n \in \mathbf{Z}} \widehat{\phi_{i, k}}\left(2^{j}(\xi+2 n \pi)\right) \overline{\hat{\psi}(\xi+2 n \pi)}
$$

converges absolutely a.e. In view of Eqs. (2) and (4) we get

$$
\begin{gathered}
\sum_{n \in \mathbf{Z}} \widehat{\phi_{i, k}}\left(2^{j}(\xi+2 n \pi)\right) \overline{\widehat{\psi}(\xi+2 n \pi)} \\
=\sum_{p=1}^{a_{i}} \mu_{k, p}^{i}\left(2^{j} \xi\right)\left[\sum_{n \in \mathbf{Z}} \widehat{\psi}\left(2^{j_{p}+j}(\xi+2 n \pi)\right) \overline{\widehat{\psi}(\xi+2 n \pi)}\right]=0 \quad \text { a.e. }
\end{gathered}
$$

Therefore all $\widehat{\phi_{i, k}}$ belong to $\sum_{j \geq 1} \oplus \widehat{W_{-j}}$.

Now it is easy to see that the integral translations of $\phi_{i, k} i \in L, k=1,2, \ldots, a_{i}$, form a tight frame with frame constant 1 for the subspace $V_{0}:=\sum_{j>1} \oplus W_{-j}$. If we set $V_{j}=D^{j}\left(V_{0}\right), j \in \mathbf{Z}$, it is not hard to verify that we get a GFMRA of $L^{2}(\mathbf{R})$ and that $\psi$ is an orthonormal wavelet associated with this type of multiresolution structure, in the sense that $\{\psi(x-k): k \in \mathbf{Z}\}$ is an orthonormal basis for the subspace $V_{1} \cap V_{0}^{\perp}$.

An interesting application of the previous proof is the following corollary:

Corollary 7. Let $\psi$ be an orthonormal wavelet and $P_{0}$ be the orthogonal projection onto $\sum_{j \geq 1} \oplus W_{-j}$. Then for every $f, g$ in $L^{2}(\mathbf{R})$ we have that

$$
\left\langle P_{0} f, g\right\rangle=\frac{1}{2 \pi} \int_{-\pi}^{\pi}\langle P(\xi) X f(\xi), X g(\xi)\rangle d \xi
$$

where $P(\xi)$ is the orthogonal projection defined onto the subspace $\left[\Psi_{j}(\xi): j \in \mathbf{Z}^{+}\right]^{-}$, $\xi \in \mathbf{T}$.

Proof. Due to polarization identity it is enough to prove the previous identity for $f=g$. Since the integral translations of $\phi_{i, k}, i \in L, k=1,2, \ldots, a_{i}$, form a tight 
frame with frame constant 1 for the subspace $V_{0}:=\sum_{j \geq 1} \bigoplus W_{-j}$, we have that

$$
\begin{aligned}
\left\|P_{0} f\right\|^{2} & =\frac{1}{(2 \pi)^{2}} \sum_{i \in L} \sum_{k=1}^{a_{i}} \sum_{l \in \mathbf{Z}}\left|\int_{-\infty}^{\infty} f(\xi) \widehat{\widehat{\phi_{i, k}}(\xi)} e^{i l \xi} d \xi\right|^{2} \\
& =\frac{1}{2 \pi} \sum_{i \in L} \sum_{k=1}^{a_{i}} \int_{-\pi}^{\pi}\left|\sum_{n} f(\xi+2 n \pi) \overline{\widehat{\phi_{i, k}}(\xi+2 n \pi)}\right|^{2} d \xi \\
& =\frac{1}{2 \pi} \sum_{i \in L} \sum_{k=1}^{a_{i}} \int_{-\pi}^{\pi} \chi_{I_{i}}(\xi)\left|\left\langle X f(\xi), \Phi_{i, k}(\xi)\right\rangle\right|^{2} d \xi \\
& =\frac{1}{2 \pi} \sum_{i \in L} \int_{-\pi}^{\pi} \chi_{I_{i}}(\xi)\|P(\xi) X f(\xi)\|^{2} d \xi \\
& =\frac{1}{2 \pi} \int_{-\pi}^{\pi}\|P(\xi) X f(\xi)\|^{2} d \xi .
\end{aligned}
$$

This completes the proof.

We close with an application of the techniques of the previous proof on Journé's example. Let $K=[-32 \pi / 7,-4 \pi) \cup[-\pi,-4 \pi / 7) \cup[4 \pi / 7, \pi) \cup[4 \pi, 32 \pi / 7)$. It can be proved that $\chi_{K}$ is the Fourier transform of an orthonormal wavelet. For this particular wavelet the dimension function is given by

$$
D_{\psi}(\xi)= \begin{cases}1, & 6 \pi / 7 \leq|\xi|<\pi \\ 0, & 4 \pi / 7 \leq|\xi|<6 \pi / 7 \\ 1, & 2 \pi / 7 \leq|\xi|<4 \pi / 7 \\ 2, & 0 \leq|\xi|<2 \pi / 7 .\end{cases}
$$

Set $I_{1}=\{\xi: 2 \pi / 7 \leq|\xi|<4 \pi / 7$ or $6 \pi / 7 \leq|\xi|<\pi\}$ and $I_{2}=[-2 \pi / 7,2 \pi / 7)$. Following the proof of the announced result we have $I_{2,1}=[-2 \pi / 7,2 \pi / 7)$ and

$$
\Psi_{1}(\xi)= \begin{cases}\delta_{-1, k}, & -2 \pi / 7 \leq \xi<0 \\ \delta_{1, k}, & -0 \leq \xi<2 \pi / 7\end{cases}
$$

For $\xi \in I_{2,1, m+4}=\left[\frac{-2 \pi}{2^{m} \cdot 7}, \frac{-\pi}{2^{m+2}}\right) \cup\left[\frac{\pi}{2^{m+2}}, \frac{2 \pi}{2^{m} \cdot 7}\right), m \geq 0$, we have that $\left\{\Psi_{1}(\xi)\right.$, $\left.\Psi_{m+4}(\xi)\right\}$ is an orthonormal basis of $\left[\Psi_{j}(\xi): j \in \mathbf{Z}^{+}\right]^{-}$. Notice that $\Psi_{m+4}(\xi)=$ $\delta_{0, k}$. For $\xi \in I_{2,1, m}=\left[\frac{-\pi}{2^{m}}, \frac{-2 \pi}{2^{m-1} \cdot 7}\right) \cup\left[\frac{2 \pi}{2^{m-1} \cdot 7}, \frac{\pi}{2^{m}}\right), m \geq 2$, we have that $\left\{\Psi_{1}(\xi)\right.$, $\left.\Psi_{m}(\xi)\right\}$ is an orthonormal basis of $\left[\Psi_{j}(\xi): j \in \mathbf{Z}^{+}\right]^{-}$and $\Psi_{m}(\xi)=\delta_{0, k}$. Therefore in the first case we have

$$
\Phi_{2,1}(\xi)=\Psi_{1}(\xi) \quad \text { and } \quad \Phi_{2,2}(\xi)=\Psi_{m+4}(\xi),
$$

while in the second case

$$
\Phi_{2,1}(\xi)=\Psi_{1}(\xi) \quad \text { and } \quad \Phi_{2,2}(\xi)=\Psi_{m}(\xi)
$$

It can be proved that $\widehat{\phi_{1,1}}=\chi_{E}$, where $E=[-8 \pi / 7,-\pi) \cup[-4 \pi / 7,-2 \pi / 7) \cup$ $[2 \pi / 7,4 \pi / 7) \cup[\pi, 8 \pi / 7), \widehat{\phi_{2,1}}=\chi_{[-16 \pi / 7,-2 \pi) \cup[2 \pi, 16 \pi / 7)}$ and $\widehat{\phi_{2,2}}=\chi_{[-2 \pi / 7,2 \pi / 7)}$. Following [4] the sets of the integral translates of $\phi_{2,1}$ and $\phi_{2,2}$ are tight frames but not disjoint, because $[0,2 \pi / 7)+2 \pi=[2 \pi, 16 \pi / 7)$. Notice that the intervals whose union is the set $E$ are not translates by integral multiples of $2 \pi$ of any other of these intervals or the intervals used in the formulas giving $\widehat{\phi_{2,1}}$ and $\widehat{\phi_{2,2}}$.

This result was produced during the author's visit in College Station, Texas, for the Linear Analysis and Probability Workshop organised by the Department 
of Mathematics of Texas A\&M University during August 1997. It was announced first to Professor D.R. Larson and his PhD. student D. Han and a few days later the proof was completed and the result was presented to Professor G. Weiss of Washington University in St. Louis. Finally we must mention that our proof was inspired by Wang's proof of Theorem 2

\section{ACKNOWLEDGEMENT}

The author wishes to thank Professors D. R. Larson and G. Weiss for their constant encouragement and support. This research was financially supported by Southwestern Bell and the Linear Analysis and Probability Workshop. I would also like to thank the referee to whom the manuscript was assigned for review by the Editors of the Electronic Research Announcements of the American Mathematical Society for suggesting Corollary 7 to me.

Added in Proof. After the submission of the revised version of the present paper, a preprint ([3]) was brought to our attention proving a similar result to ours.

\section{REFERENCES}

[1] P.Auscher, Solution of two problems on wavelets, The J. Geometric Anal. $5 \mathrm{nr} 2$, pp. 181-236, 1995. MR 96g:42016

[2] J. Benedetto, S. Li, The theory of multiresolution analysis frames and applications to filter banks, Appl. Comput. Harmon. Anal. 5 (1998), no. 4, 380-427. CMP 99:02

[3] L.W. Baggett, H.A. Medina, K.D. Merrill, Generalized multiresolution analyses and a construction procedure for all wavelet sets in $\mathbf{R}^{n}$, preprint, 1998.

[4] D. Han, D.R. Larson, Frames, bases and group representations, to appear Memoirs AMS.

[5] E. Hernández, G. Weiss, A first course on wavelets, CRC Press, Boca Raton FL, 1996. MR 97i:42015

[6] G. Gripenberg, A necessary and sufficient condition for the existence of a father wavelet, Studia Mathematica 114(3), pp.207-226, 1995. MR 96d:42049

[7] X. Wang, The study of wavelets from the properties of their Fourier transforms, Ph.D. Thesis, Washington University in St. Louis, 1995.

Department of Informatics, University of Athens, Panepistimiopolis, GR-15784 ZoGrafou, Greece

E-mail address: mpapad@di.uoa.gr

Current address: Department of Mathematics, University of Houston, Houston, Texas 772043476

E-mail address: mpapadak@math.uh.edu 